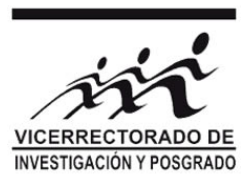

\title{
Aplicación del Algoritmo de Redes Elásticas en imágenes satélitales
}

\author{
Erick Príncipe $^{* 1,2}$ y Bram Willems ${ }^{1}$ \\ 1 Universidad Nacional Mayor de San Marcos, Ap. Postal 14-0149, Lima, Perú \\ ${ }^{2}$ Instituto Geofísico del Peru, Calle Badajoz No 169 Urb. Mayorazgo IV Etapa Ate, Lima 15012 - Perú.
}

Recibido 16 mayo 2018 - Aceptado 07 julio 2018

Esta investigación se realizó en el Santuario Nacional Los Manglares de Tumbes (SNLMT), ubicado en el distrito de Zarumilla departamento de Tumbes, está orientada a implementar una metodología que permita caracterizar la cobertura de manglar. Para ello, se analizó y procesó la imagen del sensor TM del satélites LandSat 5 evaluando una serie de parámetros relacionados a la superficie del suelo, tales como SAVI (Índice de vegetación ajustado al suelo), NDVI (Índice de vegetación de diferencia normalizada) y NDWI (Índice de agua de diferencia normalizada) con miras a establecer el índice óptimo que permita discriminar las diferentes componentes de cobertura de suelo del Santuario. El indice óptimo (SAVI) antes descrito fue introducido en el Algoritmo de las Redes Elásticas (ENA, por sus siglas en ingles) para la clasificación de la cobertura de suelo del SNLMT. Las imágenes construidas a partir de los resultados ENA, fueron sometidos al proceso de validación empleando métodos convencionales como el algoritmo de máxima verosimilitud (AMV). Tal proceso de validación consistió en realizar los análisis y comparaciones de las gráficas de firmas espectrales promedio de cada clase informacional obtenidos tanto con ENA y AMV, dando como resultados similares gráficas donde el RMSE fue por debajo de 0.052 (adimensional) y el factor de correlación sobre $r=0.886$. Esto indica que el método ENA resulta ser una herramienta eficaz para la subdivisión de clases de cobertura manglar.

Palabras claves: ENA, índice físico, máxima verosimilitud, cluster.

\section{Aplication of Elastic Net Algorithm in satellite images}

This investigation was carried out in the Santuario Nacional Los Manglares de Tumbes (SNLMT), located in the district of Zarumilla department of Tumbes, is oriented to implement a methodology that allows to characterize the mangrove cover. For this, the image of the TM sensor of the LandSat 5 satellites was analyzed and processed evaluating a series of parameters related to the soil surface, such as SAVI (Index of vegetation adjusted to the ground), NDVI (Index of vegetation of difference normalized) and NDWI (Normalized difference water index) with a view to establishing the optimum index that allows to discriminate the different components of the Sanctuary's soil cover. The optimal index (SAVI) described above was introduced in the Elastic Net Algorithm (ENA) for the classification of the SNLMT ground cover. The images constructed from the ENA results were subjected to the validation process using conventional methods such as the maximum likelihood algorithm (MLA). This validation process consisted of performing the analyzes and comparisons of the average spectral signature graphs of each informational class obtained with both ENA and MLA, resulting in similar graphs where the RMSE was below 0.052 (dimensionless) and the Correlation factor on $r=0.886$. This indicates that the ENA method proves to be an effective tool for the subdivision of mangrove coverage classes.

Keywords: ENA, physical index, maximum likelihood, cluster.

*eprincipe17@gmail.com 


\section{Introducción}

La teledetección por satélite según Copin et al. [1] es una herramienta que permite visualizar y evaluar los cambios sutiles durante un largo período de tiempo, así como la identificación de los cambios bruscos debido a los impactos antropogénicos y naturales a las áreas de bosque manglar, por ejemplo, la destrucción por un tsunami o la conversión a granjas camaroneras. Alatorre et al. [2] identificaron áreas de manglar por teledección usando imágenes LandSat en el noroeste de Mexico, mediante métodos de clasificación supervisada, reportando la discriminación entre áreas de manglar y no manglar con un umbral binario. Long y Giri [3] realizaron un mapa de distribución espacial y la extensión del área de la cobertura de bosque manglar en Filipinas alrededor del año 2000, utilizando datos imagen del sensor TM LandSat 5 para cartografiar la extensión total y la distribución espacial mediante la clasificación no supervisada. También Blasco et al. 4] realizaron un análisis espectral de imagen del sensor TM de algunas especies de mangle a nivel local. Estudios de de la cobertura manglar en la región peruana empleando sensoramiento remoto incluye la de Llactayo [5], que realiza un procesamiento digital y comprobación de campo con fines de clasificación temática y producción de mapas de los tipos de cobertura vegetal y uso de la tierra en el Santuario Nacional Los Manglares de Tumbes para los años 1982, 1983, 1992, 1996, 2001 y 1997. Asimismo, Chaccha [6] monitoreó los cambios en la cobertura y uso de la tierra en el ecosistema manglar de Tumbes en el intervalo de tiempo de 1982-1992. Asimismo, Cuya [7] realizó un diseño de criterios para clasificación de los ecosistemas de la zona del manglar de Tumbes mediante interpretación visual de imágenes MSS y SPOT.

Por los estudios del manglar anteriormente mencionados, tanto en el Perú como en el mundo una tarea importante constituye la validación de las imágenes de satélite como instrumento para el estudio, vigilancia y la evaluación de eventos naturales que podría afectar a dicho ecosistema. El presente trabajo aborda la evaluación de un procedimiento de subdivisión de clases a saber, el Algoritmo de Redes Elástica (ENA, por sus siglas en ingles), el cual fue formulado por Durbin-Willshaw [8] como un método heurístico, para resolver el "problema del comerciante viajero"(Traveling Salesman Problem-TSP). Por su parte, Rose et al. [9] le dieron una formulación mecánico-estadístico y fue aplicado entre otros, en imágenes médicas (tomograma) para la detección del tumor cancerígeno y su tamaño 10. En un reciente trabajo realizado en el Laboratorio de Teledetección de la Facultad de Ciencias Físicas de la Universidad Nacional Mayor de San Marcos [11] se empleó ENA como método para la detección automatizada de los glaciares en las imágenes de satélite, lo que ha demostrado su eficacia en la identificación y discriminación de áreas glaciares versus las no glaciares, así como los diferentes ti- pos de glaciares presentes.

El objetivo de esta investigación es implementar una metodología para la clasificación de la cobertura de manglar del SNLMT empleando imágenes LandSat, para lo cual se plantean algunos objetivos específicos que consisten en: establecer índices óptimos para el estudio de la cobertura en el SNLMT mediante imágenes procedente del satélite LandSat y evaluar la capacidad del algoritmo de las redes elásticas como un método de clasificación de la cobertura de suelo empleando imágenes del satélite LandSat.

\section{Fundamento teórico}

\section{1. Índices físicos}

Los índices físicos son calculados a partir de valores de reflectividad a distintas longitudes de onda, con el fin de extraer la información relacionada con la vegetación y el vapor de agua, minimizando los efectos de los otros factores externos como las propiedades ópticas del suelo, la irradiancia solar y la atmosférica [12]. Además, permite reducir el número de dimensiones propias de las medidas multiespectrales a una sola dimensión. Así, los índices NDVI (Ec 1) y SAVI (Ec 2) se basan en las características espectrales únicas bien identificadas de la vegetación saludable sobre las longitudes de onda del visible al infrarrojo.

$$
\begin{gathered}
N D V I=\frac{\rho_{\text {nir }}-\rho_{r}}{\rho_{\text {nir }}+\rho_{r}} \\
S A V I=\frac{\left(\rho_{\text {nir }}-\rho_{r}\right)}{\left(\rho_{\text {nir }}+\rho_{r}+L\right)}(1+L)
\end{gathered}
$$

Donde :

$\rho_{r}: \quad$ Reflectividad en la banda del rojo.

$\rho_{\text {nir }}$ : Reflectividad en la banda del infrarrojo cercano.

$\mathrm{L}$ : $\quad$ Factor de corrección, asume el valor 0 para una cubierta de vegetación muy alta, 1 para una cubierta muy baja y 0,5 para la cubierta intermedia [13.

Al igual que NDVI el SAVI proporciona la medida relativa del verdor de la vegetación a partir del contraste espectral que se produce entre las bandas del infrarrojo y el rojo. Po otra parte, el indice NDWI (Ec 3) fue desarrollado para determinar el contenido de agua en la vegetación [14] y de este modo cuantificar la cantidad de agua existente en la cubierta vegetal o el nivel de saturación de humedad que posee el suelo 15 .

$$
N D W I=\frac{\rho_{\text {nir }}-\rho_{\text {swir }}}{\rho_{\text {nir }}+\rho_{\text {swir }}}
$$

Donde :

$\rho_{\text {nir }}$ : Reflectividad en la banda del infrarrojo cercano.

$\rho_{\text {swir }}$ : Reflectividad en la banda del Infrarrojo de onda corta. 


\subsection{Algoritmo de las redes elásticas}

El algoritmo de las redes elásticas es un método heurístico de agrupamiento de datos formulado por Durbin y Willshaw [8] y reformulado con fundamentos mecánicoestadístico por Rose et al. [9], lo cual es mostrado en las ecuaciones siguientes. Para un número dado de punto de datos $x_{i}$ en el espacio n-dimensional $\left(x_{i} \in R^{n}\right)$, se define una cadena de nodos $y_{j}$ donde, los nodos representan cuentas de subconjuntos que agrupan al total de los datos con los que se cuenta. Las variables $x_{i}, y_{j}$ están relacionados entre sí por la función de energía distancia cuadrado.

$$
E_{i j}=\frac{1}{2}\left|x_{i}-y_{j}\right|^{2}
$$

Los datos y los nodos están normalizados y sin dimensiones. La energía total está dada por [16]:

$$
E=\sum_{i} \sum_{j} P_{i j} E_{i j}
$$

Con una distribución de probabilidad $P_{i j}$ desconocida. Para encontrar la distancia $\left|x_{i}-y_{j}\right|$, se pone el sistema en contacto con una fuente de calor y utilizando el principio de máxima entropía (recordando que este método es heurístico, esto implica resolver el problema de una forma no cotidiana, para este caso se considera a la máxima entropía como un principio que establece a la distribución de probabilidad menos sesgada que se le puede atribuir a un sistema estadístico; es decir aquella en la que dadas unas ciertas condiciones fijas se maximiza la entropía, esto es, aquella en la que la desinformación es máxima) [17, 18. se encuentra con la restricción de la ecuación. (5) de una distribución de probabilidad Gaussiana 9 16]:

$$
P_{i j}=\frac{e^{-\beta E_{i j}}}{Z_{i}}
$$

con la función de partición para el punto de datos $i$

$$
Z_{i}=\sum_{j} e^{-\beta E_{i j}}
$$

Para un conjunto dado de clusters, se supone que las probabilidades relativas de $i$ son independientes para cada nodo. De ahí se encuentra la función de partición total:

$$
Z=\prod_{i} Z_{i}
$$

Y la correspondiente energía libre de Helmholtz es 16 19]:

$$
F=-\frac{1}{\beta} \ln Z+E_{n o d}=-\frac{1}{\beta} \sum_{i} \ln \sum_{j} e^{-\beta E_{i j}}+E_{n o d}
$$

Donde $E_{\text {nod }}=\frac{1}{2} \lambda \sum_{j}\left|y_{j}-y_{j-1}\right|^{2}$ se añade como una energía de interacción entre los nodos [10], la cual se comporta como la energía de un resorte de constante de elasticidad $\lambda$. Al minimizar la energía libre con respecto a las posiciones de los nodos, proporciona para cada valor de $\beta$ una ecuación no lineal acoplada para las posiciones de nodo óptimas (Ec.10):

$$
\sum_{i} P_{i j}\left(x_{i}-y_{j}\right)+\lambda\left(y_{j+1}-2 y_{j}+y_{j-1}\right)=0, \forall j
$$

La ecuación (10) se resuelve para diferentes valores de $\beta$ iterativamente mediante el algoritmo de máxima pendiente el cual resulta en [20]:

$$
\begin{aligned}
\triangle y_{j} & =-\triangle \tau \frac{\partial F}{\partial y_{i}} \\
& =\triangle \tau \sum_{j} P_{i j}\left(x_{i}-y_{j}\right)+\triangle \tau \lambda\left(y_{j+1}-2 y_{j}+y_{j-1}\right) ; \forall j .
\end{aligned}
$$

Donde el paso de tiempo $\triangle \tau$ es elegido adecuadamente. En general, tendremos un gran número de puntos de datos que interactúan con un reducido número de nodos, y para una constante de elasticidad $(\lambda)$ no tan grande [10], la energía de interacción del nodo será pequeño con respecto a la energía de interacción entre los nodos y puntos de datos; esto puede ser tratado como una perturbación. Con el fin de preservar la topología de la cadena, se utiliza la interacción nodo-nodo. Si se escribe la ecuación (10) de la forma:

$$
\frac{\sum_{i} P_{i j} x_{i}}{\sum_{i} P_{i j}}-y_{j}+\frac{\lambda}{\sum_{i} P_{i j}}\left(y_{j+1}-2 y_{j}+y_{j-1}\right)=0 \quad ; \quad \forall j
$$

Podemos notar que el segundo término $\frac{\lambda}{\sum_{i} P_{i j}} \ll 1$ de la ecuación (12) es despreciable. Esto es, si casi todos los nodos $y_{i}$, son suficientes puntos de datos que contribuyen con gran probabilidad, y por el contrario, si hay pocos datos que contribuyen, entonces, la vecindad mas próxima entre nodos debe estar presente a una distancia más pequeña y de nuevo el segundo término es despreciable debido a la pequeña diferencia entre los nodos. Esto significa que en una buena aproximación la ecuación (12) se puede reducir a:

$$
y_{i}=\sum_{i} F_{i j} x_{i} \quad, \quad \forall j
$$

que representan a los centros de masa óptimos de los clusters para un conjunto de distribuciones de probabilidades asociada a los puntos de datos. A estas probabilidades se las denominan pertenencia difusa en grupos fuzzy membership in clusters 99. Para nuestro caso los denotaremos como fuzzy clusters (grupos difusos) para estas agrupaciones. 
Por tanto para cada nodo, tenemos la distribución de probabilidad asociados para los fuzzy clusters, propuesta por:

$$
F_{i j}=\frac{P_{i j}}{\sum_{i} P_{i j}}
$$

\subsection{Algoritmo de máxima verosimilitud}

El algoritmo de máxima verosimilitud es un método de clasificación, donde se utiliza el modelo probabilístico con una distribución gaussiana para formular sus reglas de decisión en la categorización de los pixeles. Los parámetros necesarios para el modelo, como la media y la matriz de covariancia se obtienen de los datos de entrada 21.

Dada la probabilidad $p\left(\omega_{i} \mid \mathbf{x}\right)$ de encontrar un pixel de clase $\omega_{i}$ en la posición $\mathbf{x}$, se tendrá una probabilidad desconocida, $p\left(\omega_{i} \mid \mathbf{x}\right)$, que es la que interesa y una desconocida $p\left(\mathbf{x} \mid \omega_{i}\right)$ obtenible a partir de los datos de entrada lo cual está relacionado por el teorema de Bayes, la probabilidad de que un píxel de cualquier clase se pueda encontrar en la posición $\mathrm{x}$ claramente es la suma de las probabilidades de que los píxeles se encuentren allí de todas las clases disponibles. En otras palabras:

$$
p\left(\omega_{i} \mid \mathbf{x}\right)=p\left(\mathbf{x} \mid \omega_{i}\right) p\left(\omega_{i}\right) / p(\mathbf{x})
$$

Esta probabilidad se denomina a priori, pues debe ser estimada antes de la clasificación. El producto $p\left(\mathbf{x} \mid \omega_{i}\right) p\left(\omega_{i}\right)$ es Ilamado probabilidad conjunta del evento $\mathbf{x}$ y $\omega_{i}$. Esto es interpretado estrictamente como la probabilidad de que un pixel ocurra en la posición $\mathbf{x}$ dado que estamos interesados en la clase $\omega_{i}$. En cuanto a $p(\mathbf{x})$ es la probabilidad de encontrar un pixel de cualesquiera de las clases en la posición $\mathbf{x}$. La probabilidad conjunta es escrita como:

$$
p(\mathbf{x})=\sum_{i=1}^{M} p\left(\mathbf{x} \mid \omega_{i}\right) p\left(\omega_{i}\right)
$$

Para el caso de una dimensión espectral la probabilidad condicional es descrita por

$$
p\left(\mathbf{x} \mid \omega_{i}\right)=(2 \pi)^{-1 / 2} \sigma_{i}^{-1} \exp \left\{-\frac{1}{2}\left(\mathbf{x}-m_{i}\right)^{2} / \sigma_{i}^{2}\right\}
$$

Donde $\mathbf{x}$ es la única variable espectral, $m_{i}$ es el valor medio de $\mathbf{x}$ y $\sigma_{i}$ es su desviación estándar; el cuadrado de la desviación estándar, $\sigma_{i}^{2}$, se denomina varianza de la distribución. La media se conoce como el valor esperado de $\mathbf{x}$ ya que, en promedio, es el valor de lo que se observará en muchos ensayos. Se calcula como el valor medio de un gran número de muestras de $\mathbf{x}$. La varianza de la distribución normal se encuentra como el valor esperado de la diferencia al cuadrado de $x$ y su media. Un promedio simple de esta diferencia al cuadrado da una estimación sesgada. Se obtiene una estimación imparcial de:

$$
\sigma_{i}^{2}=\frac{1}{q_{i}-1} \sum_{j=1}^{q_{i}}\left(\mathbf{x}_{j}-m_{i}\right)^{2}
$$

Donde $q_{i}$ es el número de pixeles en la clase $\omega \mathrm{y} \mathbf{x}_{j}$ es la $j$-esima muestra.

\section{Datos}

El dato empleado es una imagen del sensor TM a bordo del satélite LandSat 5 cuya fecha de adquisición de terrenos es $7 / 09 / 1998$, tiene 6 bandas multiespectrales, tres en el visible $(0.45-0.9 \mu \mathrm{m})$, una en el infrarrojo próximo $(0.76-0.90 \mu \mathrm{m})$ y dos del infrarrojo medio $(1.55-1.75,2.08-2.35 \mu \mathrm{m})$, todos con resolución espacial de $30 \mathrm{~m}$ [23]. La imagen seleccionada corresponde al PATH 11 y ROW 62 con la menor cantidad de cobertura de nube, menor al $10 \%$. Para cubrir el SNLMT se requirió una escena de imagen LandSat 5, correspondiente al producto L1G que es ortorectificado en la proyección UTM.

\section{Metodología}

La metodología empleada se ha dividido en dos partes (Fig. 11). La primera parte consta de cuatro procesos, los cueles son: selección y calibración de imágenes, combinación de bandas y cálculo de índices físicos. La segunda parte comprende el proceso de obtención de las clases de cobertura de suelo mediante ENA y AMV, los cuales son validados mediante las comparaciones de las gráficas de firmas espectrales promedio de las clases, estas firmas son curvas de reflectividad espectral en función de la longitud de onda 21.

\subsection{Calibración radiometríca y atmosférica}

Para convertir los números digitales (ND) de los pixeles de la imagen TM a radiancia es necesario multiplicar el valor de ND por el factor de escala radiometrica aplicando la ecuación propuesta por Chander et al. [23. la cual emplea los datos del metadato que trae consigo la imagen TM. El resultado de aplicar dicha ecuación será la magnitud física radiancia del pixel en unidades de Watts por esterioradian por metro cuadrado (Watts $/ \mathrm{m}^{2} . \mathrm{sr} \mu \mathrm{m}$ ).

Para el cálculo del valor de reflectancia de superficie implica realizar la corrección respecto al efecto que imprime la atmósfera sobre la señal recibida por el sensor, así, si se desea obtener información sobre la superficie terrestre es necesario realizar la corrección atmosférica. Este último proceso fue realizado mediante el módulo FLAASH del software ENVI (http://www.harrisgeospatial.com), para verificar que se ha realizado un proceso correcto se observan los valores de reflectividad, los cuales deberán ir de 0 a 1. 


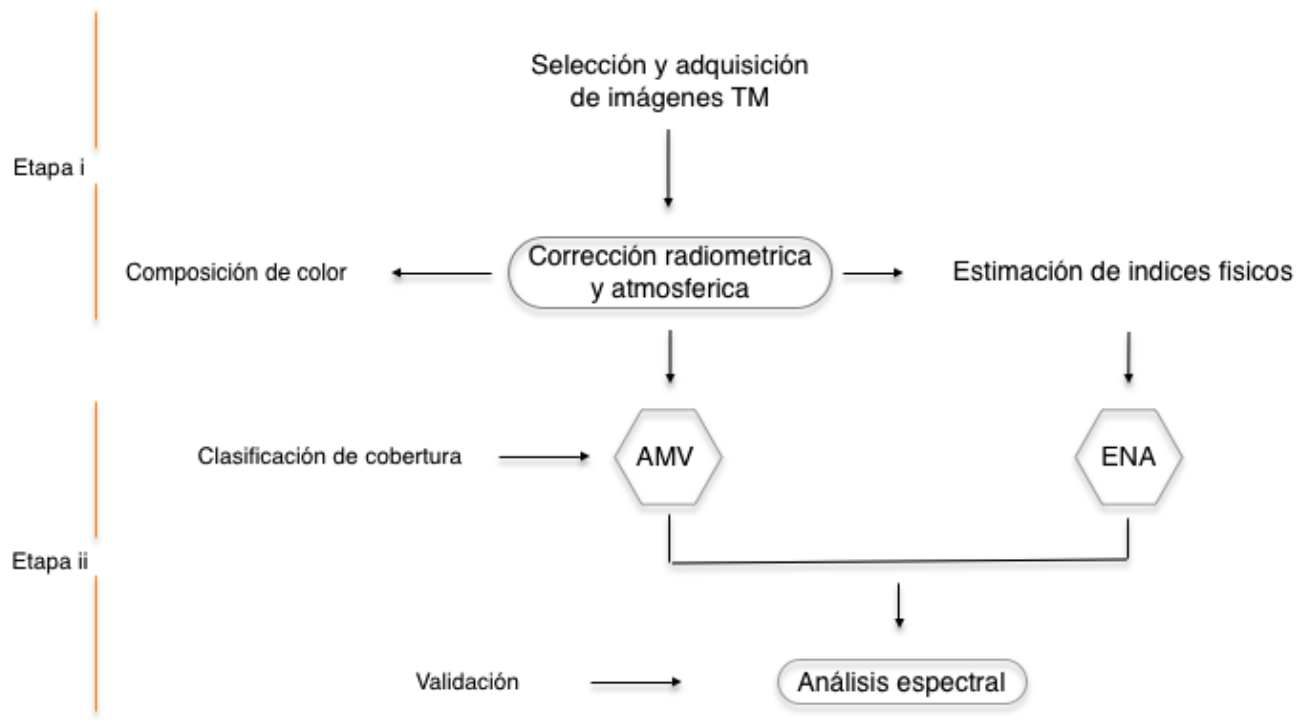

Figura 1: Diagrama de flujo de la metodología empleada en esta investigación.

\subsection{Composición de color y cálculo de indices físicos.}

Los índices físicos son transformaciones matemáticos calculados mediante las ecuaciones (1), (2) y (3) de las bandas espectrales sometidas a correcciones radiométrica y atmosféricas para un mejor resultado de análisis en comparación a los originales. Tal como indica Abdou [24], los índices tienen potencial para identificar áreas de manglar de diferente densidad y vigor.

Por otra parte, desplegando una banda por cada uno de los tres colores primarios y combinando su información espectral, rojo, verde y azul (RGB), es posible obtener una gran variedad de combinaciones que permiten extraer mayor información de la imagen. La combinación de bandas RGB: 742 permite la interpretación visual, resultando la cobertura manglar en color verde.

\subsection{Proceso de clasificación mediante AMV y ENA}

\subsubsection{AMV}

Para la clasificación supervisada se emplea el algoritmo (AMV), el cual consta de las siguientes etapas:

Análisis de separabilidad de firmas espectrales: este procedimiento permite evaluar el grado en el cual la categoría puede ser distinguida espectralmente, la separabilidad entres dos clases es considerada buena cuando el valor cerca de 1.9 , y es muy pobre cuando el valor está por debajo de 1 [25].

Matriz de confusión y cálculo del índice de fiabilidad: este procedimiento se empleó para conocer la proporción de error obtenido en los mapas.

\subsubsection{ENA}

Mediante el algoritmo de las redes elásticas se procedió al agrupamiento de datos, es decir a la generación de clases. Como primer paso, a partir del índice SAVI se generaron los datos de entrada de ENA en formato ASCII mediante el software ImageJ (https://imagej.net/Welcome), luego fueron graficados en el espacio de caracteres el cual permite observar el agrupamiento de las mismas con características similares de cobertura de suelo, asimismo esta gráfica proporciona un indicio de la cantidad de clases a escoger. Como paso siguiente se ejecutaron el algoritmo ENA dando como resultado 7 clases informacionales en formato ASCII el cual se reconstruyó en formato imagen que permitió realizar máscara de valores para el análisis de firmas espectrales.

\section{Resultados y discusión}

En esta sección se discuten los resultados obtenidos los cuales consisten en: análisis de los índices físicos, clasificación de la cobertura de suelo y comparación tanto mediante el análisis visual como de firmas espectrales de cada clase obtenida mediante ENA y MLV.

\subsection{Análisis de índices físicos}

La figura (2) muestra las imágenes en composición de color RGB: 742 y los índices físicos: NDVI, SAVI, ND$\mathrm{WI}$, cuyas características permiten diferenciar y explorar 
las coberturas de suelo presentes en la escena. A partir de estos índices se espera obtener aquel con las mejores prestaciones para la identificación de la cobertura vegetal en reemplazo de la imagen en falso color.

En base al análisis visual de las subfiguras (2p), (2k) y (2d), se observa que los índices NDWI y NDVI no permiten diferenciar de manera precisa las coberturas en la escena mostrada, donde la identificación de las especies de mangle no es clara, confundiéndose con las zonas correspondientes a los bosques secos. Sin embargo, en las imágenes del índice SAVI se visualiza una diferencia bastante marcada entre los valores correspondientes a las especies de mangle, cuerpos de agua, y bosque seco; lo cual sugiere que el índice SAVI presenta mejores prestaciones para el análisis de la cobertura vegetal en el SNLMT en comparación a los índices NDVI y NDWI.

Por otro lado, realizando el análisis cuantitativo de los índices físicos, se observa para el NDVI valores entre 0.2 y 0.4 , siendo zonas de vegetación con mayor vigor; mientras que los valores alrededor de cero representan a suelo desnudo y por último los valores negativos son aquellas zonas de cuerpos de agua. Asimismo, en el caso del índice NDWI los valores correspondientes a la zona de la cobertura vegetal están en el rango de 0.4 a 1.0 aproximadamente, donde el máximo valor fue 1.0 , los valores negativos representan a cuerpos de agua como el mar y los esteros. Finalmente, en el caso del índice SAVI los valores asociados a la cobertura vegetal están en el rango de 0.3 a 0.7 , para suelos desnudos entre 0.0 y 0.2 ; y para cuerpos de agua entre el mínimo que es -0.2 y 0 .

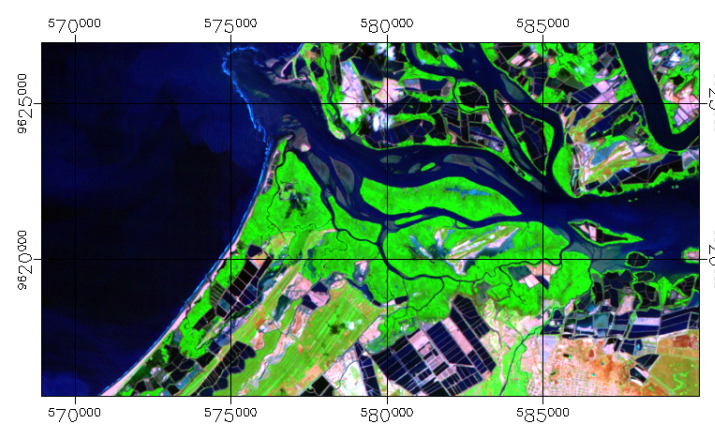

(a) RGB:742

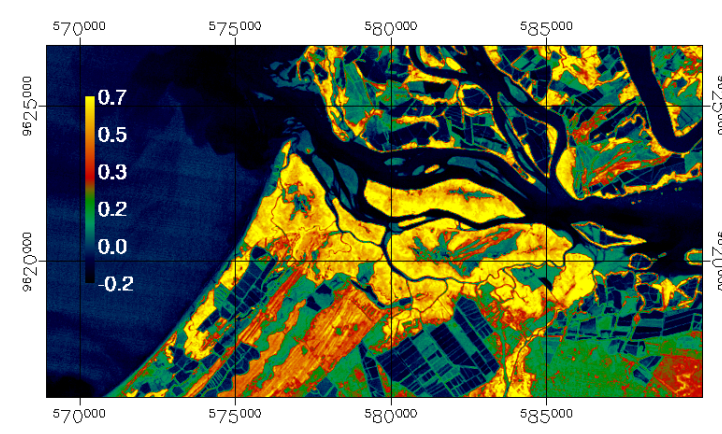

(c) SAVI

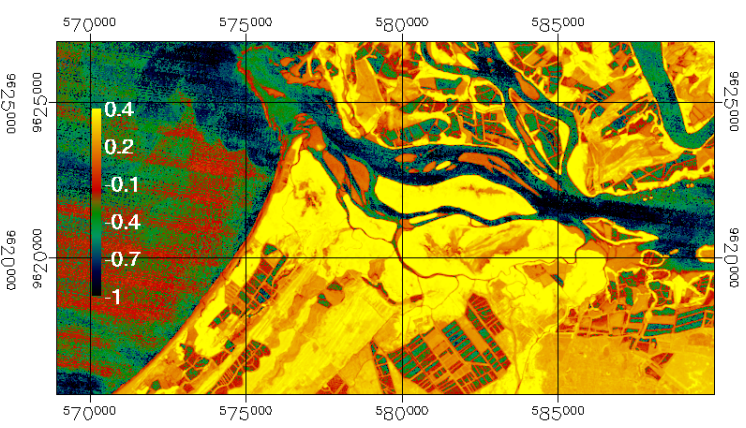

(b) NDVI

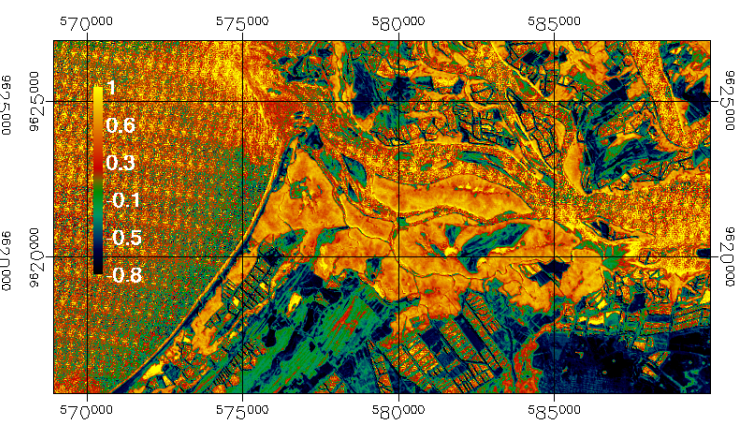

(d) NDWI

Figura 2: Imágenes en falso color RGB: 742, índices físicos NDVI, SAVI y NDWI obtenidas a partir de la imagen de reflectancia. En la figura (a), el manglar es representado en color verdusco, el suelo desnudo color blanco-pardo, cuerpos de agua en color azul que incluye los esteros, el mar y las pozas langostineras. Las imágenes de los indices físicos (b), (c) y (d) representan la cobertura vegetal para valores por encima de 0.3 ; entre 0.0 y 0.3 son zonas de suelo desnudo a cobertura vegetal rala para el caso del NDVI y SAVI, mientras para NDWI estas zonas están en intervalo de -0.5 a 0.0 . En los dos indices de vegetación los valores por debajo de cero representan a cuerpos de agua, no ocurriendo de este modo en el caso del NDWI ya que los valores cercanos a -1 representan al suelo desnudo. 
Realizado el recorte de la imagen con el polígono del límite del SNLMT (Fig. 3), se observa que los valores máximo y mínimo del indice SAVI es de 0.6 y -0.1 respectivamente. En el intervalo -0.2 a 0.0 representan a los cuerpos de agua, los valores entre $0.1-0.2$ son los suelos desnudos, y las zonas que corresponden al valor alrededor de 0.3 son aquellos zonas de bosque seco o vegetación halofita, mientras que los valores entre 0.5 a 0.6 son las zonas de cobertura manglar, donde los mayores valores son zonas de mangle de mayor tamaño en comparación a los manglares cuyos valores de SAVI están alrededor de 0.5.

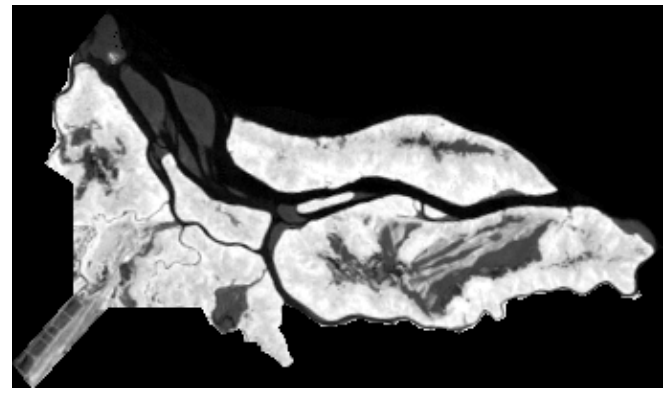

(a) SAVI- Grises

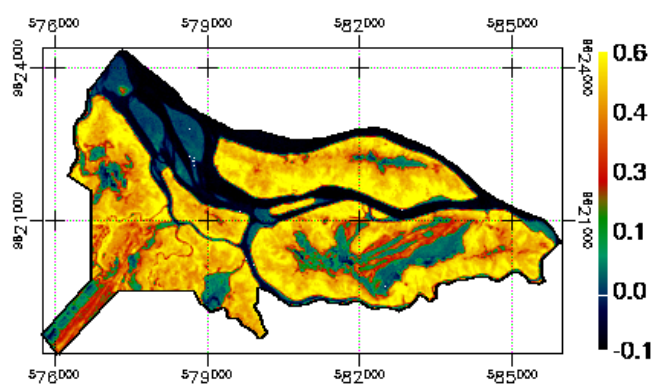

(b) Imagen SAVI

Figura 3: Imágenes del índice físico SAVI en escala de grises (a) y en barra de colores (b) de la zona de estudio-SNLMT (7/09/1998). En la figura (a) los valores más altos (0.6) corresponden al manglar de alta vigorosidad y a su vez son las zonas que se encuentran en la orilla de los esteros, mientras que los valores alrededor de 0.5 son aquellos arboles de mangle contiguos a los que se encuentra al estero. Valores entre 0.2 y 0.3 son las áreas de bosque seco, finalmente los valores en el intervalo 0.0-0.2 y los negativos representan a los suelos desnudos y cuerpos de agua respectivamente.

\subsection{Clasificación con ENA y AMV}

A continuación, se discuten los resultados de la clasificación mediante los dos algoritmos. En primer lugar para la clasificación mediante ENA se emplea como dato de entrada el índice SAVI mostrado en la figura (3), para lo cual se tomaron 7 nodos y cada uno proporciona una imagen cluster con valores similares. En segundo lugar, para aplicar el AMV se empleó como dato de entrada 7 clases se cobertura de suelo seleccionado en base al resultado proporcionado por ENA. Dichos datos de entrada son caracterizados mediante las firmas espectrales y análisis de separabilidad (distancia Jeffries-Matusita $d_{J-M}$ [25]) mostrado en la tabla (1).

Las clases de cobertura de suelo seleccionadas fueron: agua $(A)$, arena $(A r)$, vegetación dispersa $(V d)$, bosque seco (Bs), mangle tipo i (M-i), mangle tipo ii (M-ii) y mangle tipo iii (M-iii); las cuales representan a las clases obtenidas mediante AMV. En el caso de las clases mediante ENA la nomenclatura que se emplea es cluster 0 a 6; pero que se corresponden respectivamente tal como muestra la tabla de descripción de las clases (tabla 3).

A partir de la tabla (1), el valor de separabilidad más bajo corresponde a la relación entre la cobertura manglar tipo iii y i cuyo valor corresponde a separabilidad pobre (1.71), en el caso de los manglares y las clases distintas a esta los valores se sepabilidad están en el rango pobre a buena (Ver tabla 1). En la comparación entre las clases de cobertura vegetal, es decir las clases de mangle tipo i, ii y iii; bosque seco y vegetación dispersa y el agua, se aprecia en la tabla 1 valores de separabilidad sobre 1.88 esto corresponde según Marcal et al. [25] a separabilidad buena.

En las figuras (4) y (5) se muestran los resultados de la clasificación mediante ENA y AMV, donde la parte izquierda representa el resultado mediante ENA y la derecha a AMV. Se observa una coincidencia marcada de las clases agua, arena, vegetación dispersa, mangle tipo i, ii y iii; no siendo así para el caso de la clase bosque seco, ya que el cluster 3 (Fig $4 \mathrm{~g}$ ) no muestra una frontera clara entre las demás clases. Sin embargo, en un análisis general se observa que existe una buena coincidencia entre las clases obtenidas por ambos métodos, además en la clasificación mediante AMV la exactitud lograda fue alta tal como muestra la matriz de confusión (Tabla 2); donde para calcular la fiabilidad global se realiza la operación de cociente entre la traza de la matriz y la suma de los elementos de manera que el resultados de fiabilidad es del $98.65 \%$; lo cual es una evidencia más de los buenos resultados obtenidos mediante ENA. Asimismo, a partir del análisis de las firmas espectrales se puede observar alta similitud en las gráficas de la (Fig.6). 


\begin{tabular}{lcccccc}
\hline$d_{J-M}$ & $\mathrm{~A}$ & $\mathrm{Ar}$ & $\mathrm{Vd}$ & $\mathrm{Bs}$ & $\mathrm{M}-\mathrm{i}$ & $\mathrm{M}-\mathrm{ii}$ \\
\hline $\mathrm{Ar}$ & 1.99 & & & & & \\
$\mathrm{Vd}$ & 1.99 & 1.99 & & & & \\
$\mathrm{Bs}$ & 2.00 & 1.99 & 1.98 & & & \\
$\mathrm{M}-\mathrm{i}$ & 1.88 & 1.98 & 1.75 & 1.99 & & \\
$\mathrm{M}-\mathrm{ii}$ & 2.00 & 1.99 & 1.88 & 1.99 & 1.99 & \\
$\mathrm{M}-\mathrm{iii}$ & 2.00 & 2.00 & 1.99 & 1.99 & 1.71 & 1.99 \\
\hline
\end{tabular}

Tabla 1: Valores de separabilidad de las clases de cobertura de suelo.

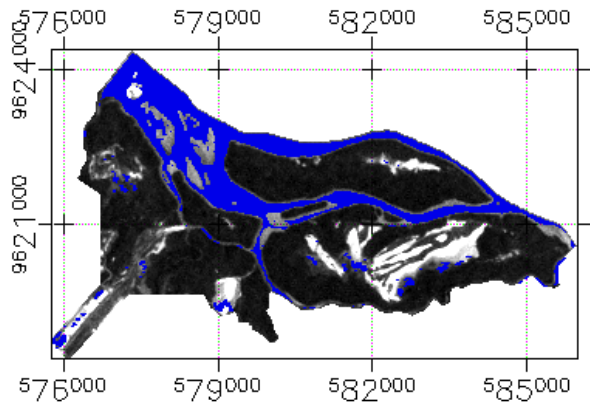

(a) Cluster 0

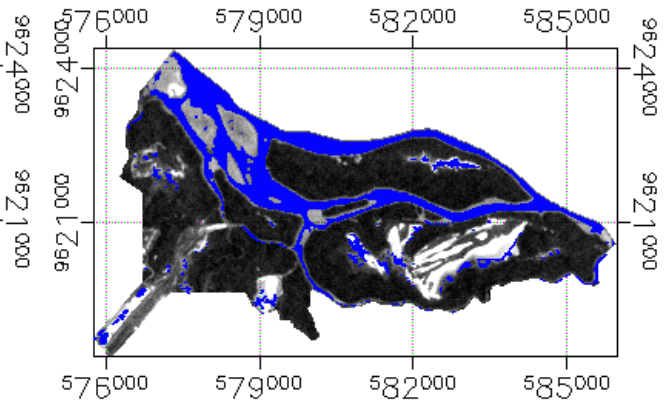

(b) Agua

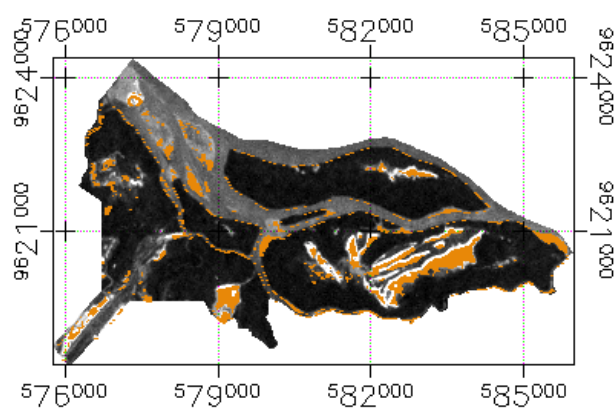

(c) Cluster 1

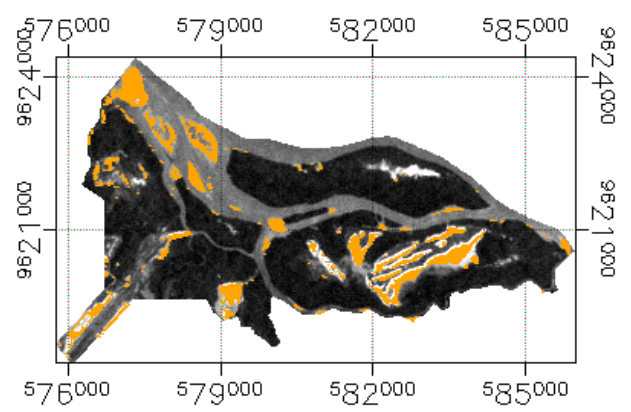

(d) Arena

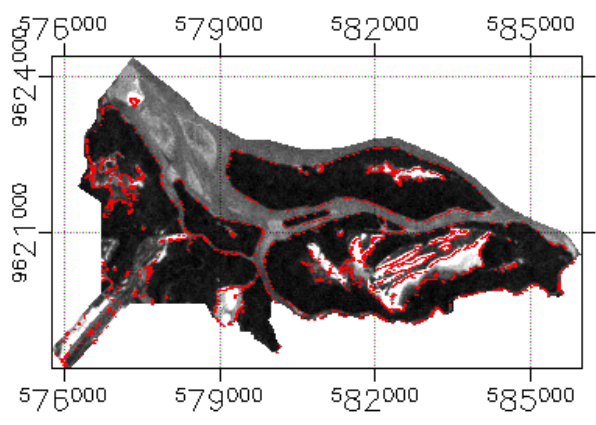

(e) Cluster 2

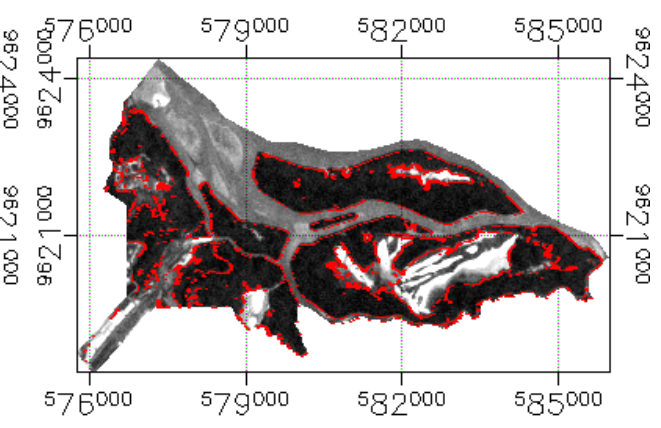

(f) Vegetación dispersa

Figura 4: Imagen de reflectancia de la banda 1 sobre la cual se resalta en colores las clases: izquierda - ENA y derecha - AMV. — agua; arena; vegetación dispersa; bosque seco 


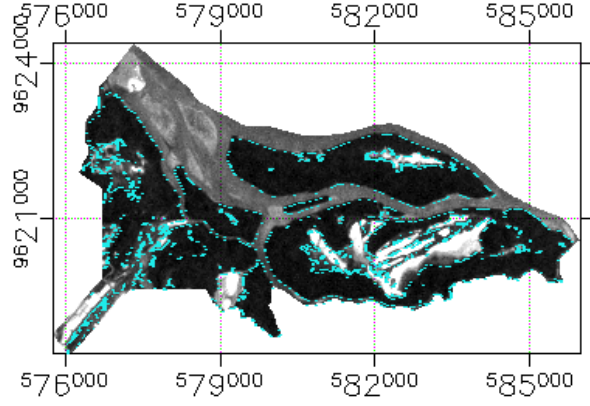

(a) Cluster 3

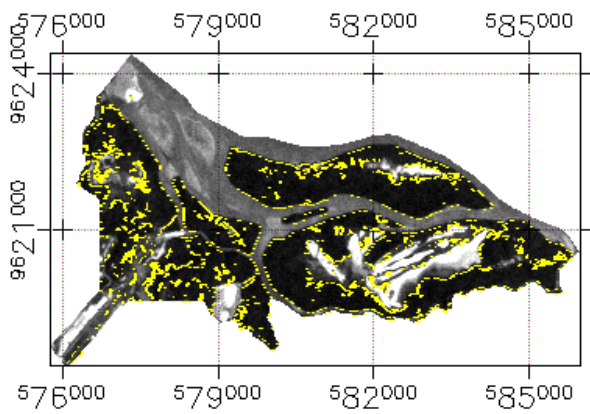

(c) Cluster 4

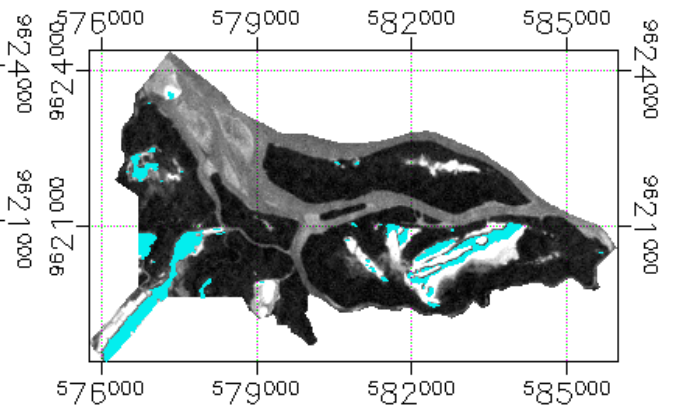

(b) Bosque seco

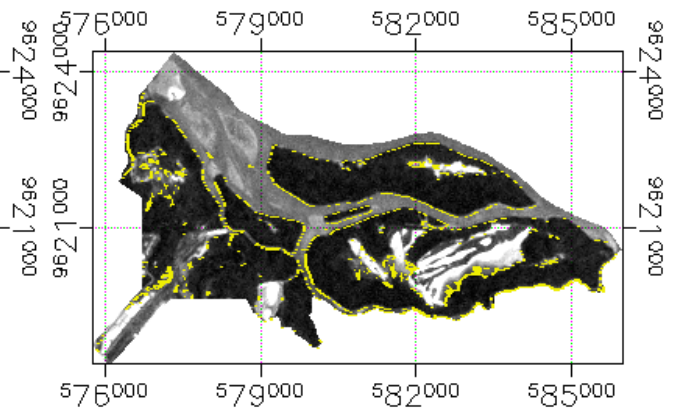

(d) Mangle tipo i

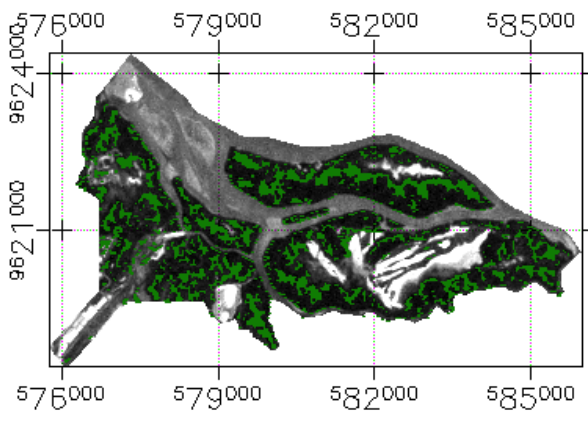

(e) Cluster 5

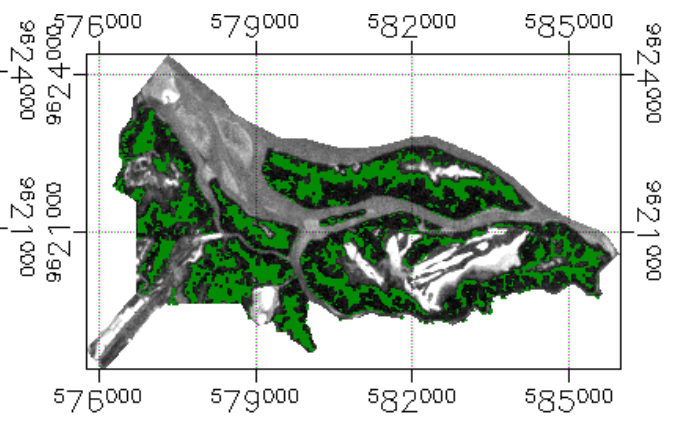

(f) Mangle tipo ii

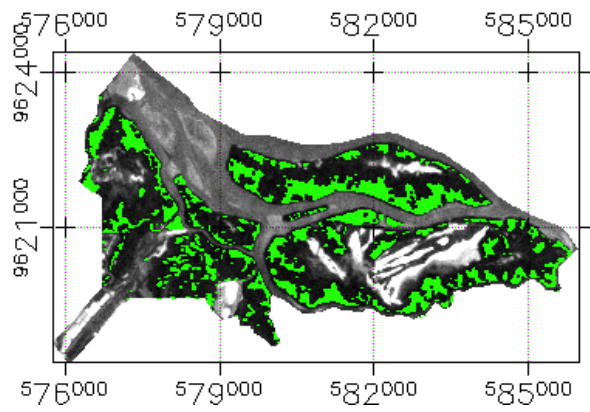

(g) Cluster 6

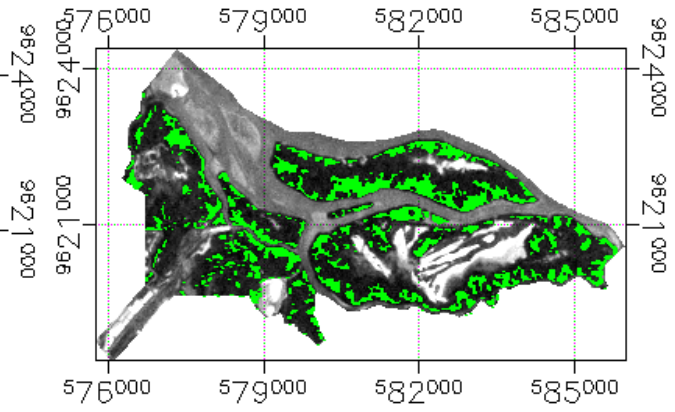

(h) Mangle tipo iii

Figura 5: Imagen de reflectancia de la banda 1 sobre la cual se resalta en colores las clases: izquierda - ENA y derecha - AMV. mangle tipo i; mangle tipo ii; mangle tipo iii 


\begin{tabular}{lcccccccc}
\hline Clase & M-iii & M-ii & M-i & Bs & Vd & Ar & A & Total \\
\hline M-iii & 203 & 1 & 0 & 0 & 0 & 1 & 0 & 205 \\
M-ii & 0 & 270 & 0 & 0 & 3 & 0 & 0 & 273 \\
M-i & 0 & 0 & 64 & 0 & 0 & 0 & 0 & 64 \\
Bs & 0 & 0 & 0 & 102 & 0 & 1 & 0 & 103 \\
Vd & 0 & 0 & 6 & 0 & 62 & 0 & 0 & 68 \\
Ar & 0 & 0 & 0 & 0 & 0 & 104 & 0 & 104 \\
A & 0 & 0 & 0 & 0 & 0 & 0 & 71 & 71 \\
\hline Total & 203 & 271 & 70 & 102 & 65 & 106 & 71 & 888 \\
\hline
\end{tabular}

Tabla 2: Matriz de confusión de clasificación supervisada mediante AMV

\begin{tabular}{ccl}
\hline Clase ENA & Clase AMV & \multicolumn{1}{c}{ Descripción } \\
\hline Cluster 0 & A & $\begin{array}{l}\text { Esta clase incluye a los cuerpos de agua salobres de los esteros y espejos de } \\
\text { agua en medio de las islas que se forma al ingresar el agua en marea alta. }\end{array}$ \\
\hline Cluster 1 & Ar & $\begin{array}{l}\text { Comprende el suelo que no posee ningún tipo de cobertura vegetal o agua, } \\
\text { el suelo de estas zonas presentan altas concentraciones de sal. Se incluye en } \\
\text { esta clase las arenas de fango que se forman cuando hay marea baja en dichas } \\
\text { zonas. Se evidencia la presencia de raíces de manglar. }\end{array}$ \\
\hline Cluster 2 & Vd & $\begin{array}{l}\text { Esta clase está conformado por salicornia fruticosa, sessuvion portolacastrum y } \\
\text { batís maritima. También comprende aquellas ramas de mangle rojo de la orilla } \\
\text { de los esteros que están inclinadas hacia los esteros. }\end{array}$ \\
\hline Cluster 3 & Bs & $\begin{array}{l}\text { Conformado por matorrales ralos, vegetación de poca vigorosidad o baja den- } \\
\text { sidad vegetal con firma espectral semejante a la vegetación en desarrollo [26]. }\end{array}$ \\
\hline Cluster 5 & M-i & $\begin{array}{l}\text { Esta clase corresponde a la especie mangle Avicenia (mangle salado). Se ob- } \\
\text { servó que este tipo de manglar se encuentran en zonas alejadas a los esteros } \\
\text { donde hay poco suministro de agua y alta concentración de sales. Esta especie } \\
\text { de mangle posee la capacidad de tolerar altas concentraciones de sal. }\end{array}$ \\
\hline Cluster 6 & M-ii & $\begin{array}{l}\text { Esta unidad comprende predominantemente a la especie de Rhrizophora mangle } \\
\text { (mangle rojo) de menores alturas en comparación a los de la orilla del estero. } \\
\text { Es la clase predominante de cobertura manglar en el SNLMT }\end{array}$ \\
\hline M-iii & $\begin{array}{l}\text { Son zonas donde se evidencia la existencia del manglar con mayor vigor y } \\
\text { tamaño, estas se encuentran en la orilla y alrededores a los esteros. La especie de } \\
\text { manglar que corresponde a estas áreas es el Rhrizophora mangle, denominado } \\
\text { mangle rojo. }\end{array}$ \\
\hline
\end{tabular}

Tabla 3: Descripción de las clases informacionales de las Figuras (4) y (5).

\subsection{Comparación de firmas espectrales de las clases obtenidas mediante ENA y AMV}

Las gráficas mostradas en la figura (6) fueron obtenidas a partir de las firmas espectrales promedio de las clases de coberturas, obtenidas mediante ENA y AMV. Se realizó la comparación de gráficas donde, las gráficas en color negro representan a las firmas espectrales de clase
ENA y las gráficas distintas a este color son las firmas espectrales promedio de las clases obtenidos por AMV. Para un mayor orden se muestra el análisis de las comparaciones de las gráficas en la tabla (5), además en la tabla (4) se indican los valores de los factores de correlación ( $r$ ) y el error cuadrático medio (RMSE, por sus siglas en ingles) los cuales permiten las comparaciones estadísticamente. 


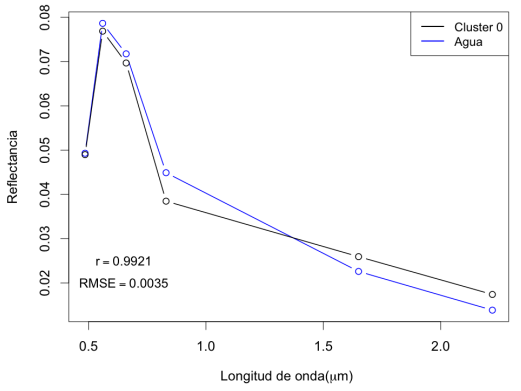

(a) Agua

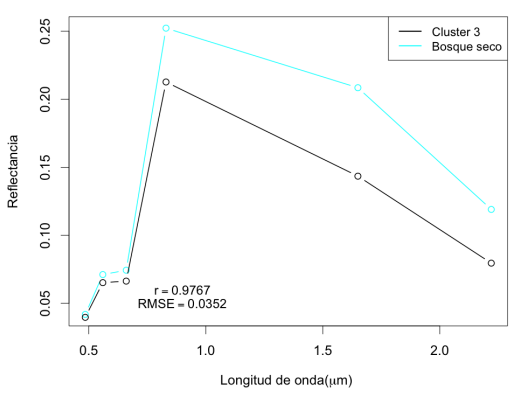

(d) Bosque seco

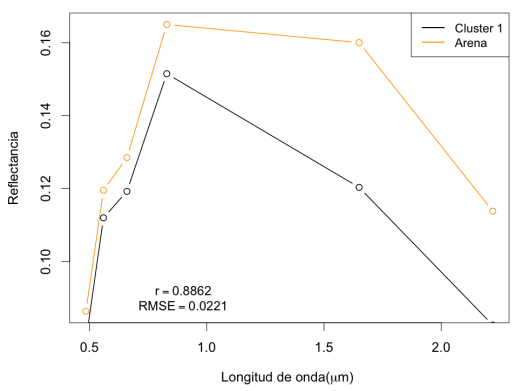

(b) Arena

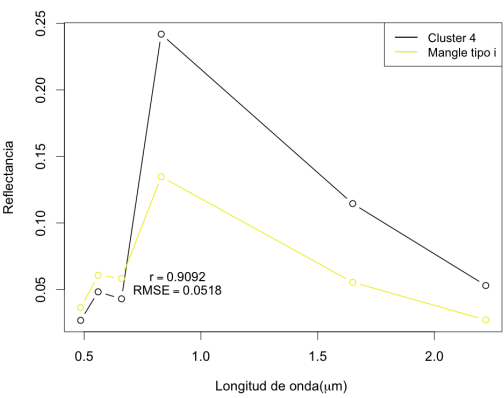

(e) Mangle tipo i

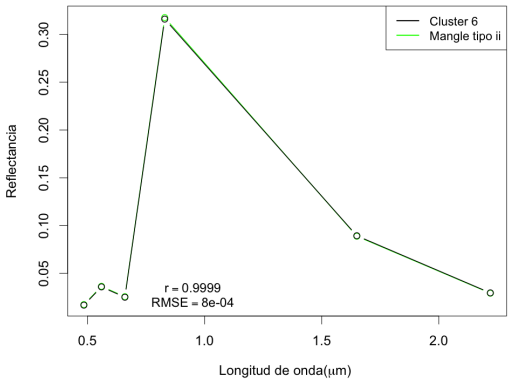

(g) Mangle tipo iii

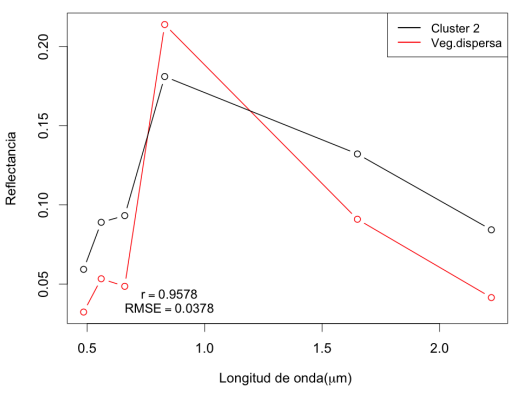

(c) Vegetación dispersa

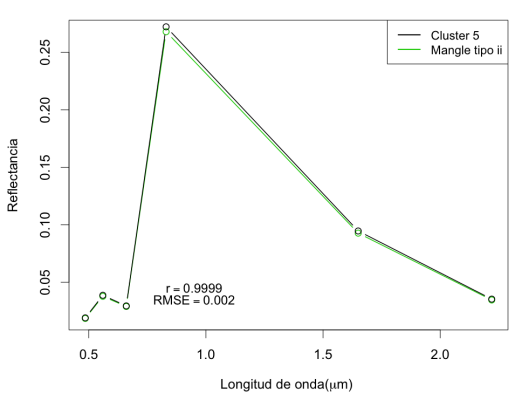

(f) Mangle tipo ii

Figura 6: Comparación de firmas espectrales de las clases: arena, agua, vegetación dispersa, bosque seco, mangle tipo i, ii y iii. Las gráficas en color negro representan a las firmas espectrales de clase ENA, mientras que las gráficas distinto al color negro representan a las firmas espectrales de la clase AMV. Además, se incorpora en la gráfica el error cudrático medio (RMSE) y el factor de correlación ( $r$ ). 


\begin{tabular}{lcr}
\hline Clases & r & RMSE (adimensional) \\
\hline Cluster 0 - A & 0.9921 & 0.0035 \\
Cluster 1 - Ar & 0.8862 & 0.0221 \\
Cluster 2 - Vd & 0.9578 & 0.0378 \\
Cluster 3 - Bs & 0.9767 & 0.0352 \\
Cluster 4 - M-i & 0.9092 & 0.0518 \\
Cluster 5 - M-ii & 0.9999 & 0.0020 \\
Cluster 6 - M-iii & 0.9999 & 0.0008
\end{tabular}

Tabla 4: Resumen de estadísticos, factor de correlación ( $r$ y error cuadrático medio (RSME) en $\mu m$.

\begin{tabular}{|c|c|}
\hline Nombre de clase & Descripción \\
\hline A & $\begin{array}{l}\text { Se observa una buena proximidad en las gráficas con valores cercanos de reflecti- } \\
\text { vidad, a excepción de las bandas } 4 \text { y } 5 \text { donde hay una ligera diferencia, mostrando } \\
\text { un } r=0.9920 \text { y un RMSE }=0.0035 \text { lo cual sugiere que gran parte de la zona de clase } \\
\text { cluster } 0 \text { coincide con la clase agua. }\end{array}$ \\
\hline Ar & $\begin{array}{l}\text { Las gráficas muestran una deferencia marcada en el valor reflectancia del infrarrojo } \\
\text { (bandas } 4,5 \text { y } 7 \text { ), siendo esto una evidencia de la diferencia entre los resultados de } \\
\text { esta clase, posiblemente debido a una intrusión de cobertura vegetal o cuerpos de } \\
\text { agua. Ademas, esta comparación mostró } r=0.8861 \text { y RMSE }=0.0221\end{array}$ \\
\hline Vd & $\begin{array}{l}\text { Existe una ligera diferencia entre las firmas espectrales, en la gráfica de cluster } 2 \text { se } \\
\text { observa un valor superior de la reflectancia para las bandas del visible, } 5 \text { y } 7 \text { la cual } \\
\text { evidencia el aporte de suelo desnudo a la cobertura de esta clase. Mientras que el } \\
\text { valor de reflectividad en la banda } 4 \text { es máxima para la gráfica vegetación dispersa. } \\
\text { El valor de } r \text { en esta comparación fue de } 0.9578 \text { y un RMSE }=0.0378 \text {. }\end{array}$ \\
\hline Bs & $\begin{array}{l}\text { En ambas gráficas se evidencia la presencia de suelo desnudo, puesto que los valores } \\
\text { de reflectividad para el infrarrojo medio están por encima de los valores correspon- } \\
\text { dientes a la cobertura vegetal. Asimismo, para la gráfica bosque seco la reflectividad } \\
\text { en el infrarrojo: cercano, medio } 1 \text { y medio } 2 \text { están por encima de la gráfica de color } \\
\text { negro, el máximo de valor corresponde al infrarrojo cercano. Sin embargo, no cam- } \\
\text { bia el patrón de la firma espectral. El r y RMSE en esta comparación fue de } 0.9766 \\
\text { y } 0.3502 \text {. }\end{array}$ \\
\hline$M-i$ & $\begin{array}{l}\text { Se observa una diferencia marcada en los valores del infrarrojo cercano y medio } \\
\text { (bandas } 4,5 \text { y } 7 \text { ), la gráfica de la firma espectral de la clase cluster } 4 \text { está por } \\
\text { encima de la clase mangle bajo, es decir la firma espectral del cluster } 4 \text { representa } \\
\text { a la vegetación más vigorosa en comparación de la firma espectral de la M-i. En la } \\
\text { comparación de factor de correlación mostró el valor de } r=0.9091 \text { y RMSE }=0.0518 \text {. }\end{array}$ \\
\hline M-ii & $\begin{array}{l}\text { En estas firmas se observan una fuerte semejanza de firmas espectrales, incluso se } \\
\text { superponen las gráficas, lo cual mostró un } r=0.9999 \text { y } \mathrm{RMSE}=0.002 \text {. Por consi- } \\
\text { guiente, en ambos métodos de clasificación se consigue reproducir el mismo patrón } \\
\text { espectral ya que se aprecia un error casi nulo y correlación muy alta. }\end{array}$ \\
\hline M-iii & $\begin{array}{l}\text { Para estas clases se observa una semejanza muy marcada proporcionando un factor } \\
\text { de correlación } r=0.9999 \text { y } R S M E=0.0008 \text {, es decir ambas clasificaciones describen } \\
\text { las mismas unidades de coberturas vegetal. El valor máximode reflectancia para } \\
\text { ambas gráficas se da en el infrarojo (banda } 4 \text { ), siendo esta de mayor valor que el } \\
\text { máximo de la gráfica de clase de } M-i i \text {. En cambio, para el caso de las otras bandas los } \\
\text { valores de reflectancia correspondientes a la clase M-iii son inferiores en comparación } \\
\text { a los de clase M-ii. }\end{array}$ \\
\hline
\end{tabular}

Tabla 5: Descripción de la comparación de las firmas espectrales por separado.

\section{Conclusiones}

Las técnicas estadísticas utilizadas permitieron obtener con alta exactitud resultados de la clasificación de la cobertura de suelo. Demostrando de esta manera que el algoritmo de las redes elásticas tiene una gran capacidad para la división de la cobertura vegetal, utilizando para ello como dato de entrada el índice físico SAVI. Por lo cual, ENA es una técnica eficiente, capaz de dividir las coberturas $\sin$ necesidad de trabajo de campo, logrando implementar una metodología para el estudio de la cobertura de suelo del Santuario Nacional Los Manglares de Tumbes mediante las imágenes LandSat.

Con relación al objetivo de establecer índices óptimos pa- 
ra el estudio de la cobertura vegetal, se determinó que a partir del análisis visual los índices NDVI y NDWI no permiten una distinción clara de las coberturas en comparación al índice SAVI, lo cual sugiere que el SAVI es un índice que ofrece mejores prestaciones que los dos índices antes mencionados. Este último índice destaca perfectamente la vegetación y proporciona una buena distinción entre las coberturas de agua, suelo desnudo y vegetación; además delimita las especies de manglar, Rhizophora mangle y Avicennia germinans conocidos como mangle rojo y negro respectivamente por la distinción de color y valor del índice, lo cual se encuentra entre -1 y 1 siendo los valores inferiores o equivalentes a cero corresponden a suelo desnudo y aquellos cercanos a 1 representan a la vegetación saludable. Por consiguiente, los valores del SAVI correspondientes a la cobertura manglar están entre 0.4 y 0.6 ; mientras que los valores de 0.3 a 0.4 podrían corresponder a la vegetación de bosque seco. Asimismo, los valores entre 0.1 a 0.3 corresponderían a suelos con vegetación rala o herbácea.

El ENA como un método de clasificación permitió hacer divisiones de coberturas de suelo con las mismas propiedades espectrales tal como se observó en las imágenes (4) y (5), lo cual proporcionó las clases informacionales descrita en la tabla (3). Asimismo, los resultado de clasificación generados con ENA han sido comparados con los resultados de la clasificación con AMV cuya exactitud fue de $98.65 \%$, lo cual permitió a partir de comparaciones gráficas concluir que las firmas espectrales son razonablemente cercanas proporcionando un RMSE por debajo de $0.052 \mu \mathrm{m}$ y factor de correlación sobre $\mathrm{r}=0.886$ tal como fue mostrado en la tabla (4) y figura (6).

\section{Agradecimientos}

Un agradecimiento al proyecto del IGP que a través del proyecto Impacto de la Variabilidad y Cambio Climático en el Ecosistema de Los Manglares de Tumbes se logró desarrollar el presente estudio. Asimismo, agradecer al Laboratorio de Teledetección (LabTel) de la UNMSM.

\section{Referencias}

[1] P. Coppin, I. Jonckheere, K. Nackaerts, B. Muys, and E. Lambin. Digital change detection methods in ecosystem monitoring. A review. Int. J. Remote Sens., 25:1565-1596 (2004).

[2] L. Alatorre, R. Sanchez-Andrés, S. Cirujano, S. Begueria and S. Sanchez. Identification of mangrove areas by remote sensing: The roc curve technique applied to the northwestern Mexico coastal zone using landsat imagery. Remote Sens, 3:1568-1583 (2011).

[3] J. Long and C. Giri. Mapping the Philippines Mangrove Forests Using Landsat Imagery. Sensors, 11:2972-2981 (2011).

[4] F. Blasco, P. Saenger and E. Janodet. Mangroves as indicators of coastal change. Catena, 27(34):167-178 (1996).

[5] L. LLactayo. Análisis de imágenes satelitales y mapeo de cobertura del santuario nacional manglares de tumbes. Technical report, MEDA. (2009).

[6] H. Chaccha Córdova. Monitoreo de los cambios en la cobertura y uso de la tierra en el ecosistema manglar de Tumbes 1982-1992. Master's thesis, Facultad de Ciencias Forestales (1999).

[7] O. Cuya Matos. Diseño de criterios para clasificación de los ecosistemas de la zona del manglar de tumbes mediante interpretación visual de imágenes mss landsat y spot. Master's thesis, Especialidad en Manejo Forestal (2000).

[8] R. Durbin and D. Willshaw. An analogue approach to the travelling salesman problem using an elastic net method. Nature, 326:689-691 (1987).

[9] K. Rose, E. Gurewitz and G. Fox. Statistical mechanics and phase transitions in clustering. Phys Rev Lett, 65:945-948 (1990).

[10] M. Levano and H. Nowak. New aspects of the elastic net algorithm for cluster analysis. Neural Comput Applic. 20(1):835-850 (2011).

[11] B. Willems, F. Toledo, J. Rojas and H. Nowak. Automatizad detection of glaciers using the elastic net algorithm. Remote Sensing of Environment (2012).

[12] C. Tucker. Red and photographic infrared linear combinations for monitoriang vegetation. Remote Sens. Environ, 8:127-150 (1979).

[13] E. Sebem. Aportaciones de la teledección en el desarrollo de un sistema meteorológico para la evaluación de los efectos del cambio climático sobre la producción de las explotaciones agrarias. PhD thesis, Escuela Técnica superior de ingenieros agrónomos Universidad Politecnica de Madrid., Departamento ingeniería cartográfica, geodesia y fotogrametría. Expresión Gráfica (2005). 
[14] B. Gao. Ndwi-A normalized difference water index for remote sensing of vegetation liquid water from space. Remote Sensing of Environment., 58:257-266 (1996).

[15] J. Jackson, M. Chen, F. Cosh, M. Li, F. Anderson, C. Walthall, P. Doriaswamy and E. Hunt. Vegetation water content mapping using landsat data derived normalized difference water index for corn and soybeans. Remote Sens. Environ., 92:475-482 (2004).

[16] L. Reichl. A modern course in statistical physical. wiley, New York (1998).

[17] G. Silviu and A. Shenitzer. The principle of maximum entropy. The Mathematical Intelligencer. 7:4248 (1985).

[18] E. Jaynes. Information theory and statistical mechanics. Phys Rev, (106:620) (1957).

[19] K. Ball, B. Erman and K. Dill. The elastic net algorithm and protein structure prediction. J Comput Chem, 23(77-83) (2002).

[20] R. Duda, P. Hart and D. Stork. Pattern classification. Wiley, New York (2001).
[21] J. Richards and J. Xiuping. Remote Sensing Digital Image Analysis An Introduction. volume 1. springer, 4ta edition (2005).

[22] J. Sobrino. Teledección. Guada Impresiones S.L.(2000).

[23] G. Chander, B. Markham and D. Helder. Summary of current radiometric calibration coefficients for landsat MSS, TM, ETM+, and eo-1 ali sensors. Re- mote Sensing of Environment, 113:893-903 (2009).

[24] M. Abdou. Evaluación de la potencialidad de los índices de vegetación para auxiliar la detección de áreas degradadas en los manglares del piqui (Brail). Sao José dos Campos, INPE (1989).

[25] A. Marcal, J. Borges, J. Gomes and P. da Costa. Land cover update by supervised classification of segmend aster image. International Journal of Remote Sensing, 26(7):1347-1362 (2005).

[26] L. Palacios, F. Paz, J. Oropeza, B. Figueroa, M. Martínez, A. Ortiz and A. Exebio. Clasificador genérico de objetos en imágenes ETM+. Agrociencia, Colegio de Postgraduados Texcoco., 40(5):613-626. (2006). 